\title{
Lifetime Neutron Fluence Analysis of the Ringhals Unit 1 Boiling Water Reactor
}

\author{
Joel A. Kulesza ${ }^{1, a}$, Jenny Roudén ${ }^{2}$, and Eva-Lena Green² \\ ${ }^{1}$ Westinghouse Electric Company LLC 1000 Westinghouse Drive, Cranberry Township, \\ PA 16066, USA \\ ${ }^{2}$ Vattenfall/Ringhals AB, 43285 Väröbacka, Sweden
}

\begin{abstract}
This paper describes a neutron fluence assessment considering the entire commercial operating history ( 35 cycles or $\sim 25$ effective full power years) of the Ringhals Unit 1 reactor pressure vessel beltline region. In this assessment, neutron $(\mathrm{E}>1.0 \mathrm{MeV})$ fluence and iron atom displacement distributions were calculated on the moderator tank and reactor pressure vessel structures. To validate those calculations, five in-vessel surveillance chain dosimetry sets were evaluated as well as material samples taken from the upper core grid and wide range neutron monitor tubes to act as a form of retrospective dosimetry. During the analysis, it was recognized that delays in characterizing the retrospective dosimetry samples reduced the amount of reactions available to be counted and complicated the material composition determination. However, the comparisons between the surveillance chain dosimetry measurements $(\mathrm{M})$ and calculated $(\mathrm{C})$ results show similar and consistent results with the linear average $\mathrm{M} / \mathrm{C}$ ratio of 1.13 which is in good agreement with the resultant least squares best estimate $(\mathrm{BE}) / \mathrm{C}$ ratios of 1.10 for both neutron $(\mathrm{E}>1.0 \mathrm{MeV})$ flux and iron atom displacement rate.
\end{abstract}

\section{Introduction}

Ringhals Unit 1 in southwest Sweden is an Asea-Atom boiling water reactor (BWR) rated at 865 MWe. The reactor core has 648 fuel bundles (3680 mm maximum active fuel height $\times \sim 150 \mathrm{~mm}$ square pitch). The stainless steel reactor internals are comprised of a $40-\mathrm{mm}$ thick moderator tank surrounding the fuel atop a 70-mm thick fuel support plate. External to the moderator tank, feedwater inlet nozzles near the top of the tank permit water into the downcomer region between the moderator tank and reactor pressure vessel (RPV) which is circulated downward, through recirculation outlet nozzles outside the vessel, and then back in recirculation inlet nozzles underneath the moderator tank.

For Ringhals Unit 1, the beltline region (as typically defined) is comprised of an upper and lower course. The upper has three rolled segments joined with axial welds (at $22^{\circ}, 195^{\circ}$, and $209^{\circ}$ ), and the lower has two rolled segments joined with axial welds (at $158^{\circ}$ and $338^{\circ}$ ). The lower course also includes the recirculation pump outlet nozzles. The upper and lower course is joined by weld W1204. Each of these materials must be considered in the overall beltline region embrittlement assessments.

Ringhals Unit 1 has been in commercial operation since January 1976 which accounts for nearly 40 years of service and this analysis was a part of the Long Term Operation (LTO) program for verification of the fitness for service for yet another 10 years. The Swedish legislation is not firmly based on a

\footnotetext{
${ }^{\text {a }}$ Corresponding author: jkulesza@umich.edu
} 
Table 1. Summary of Analyzed Surveillance Chains and Retrospective Dosimetry Samples.

\begin{tabular}{|c|c|c|c|c|c|c|}
\hline Component & ID & Angle & Radius & Elevation & Date Withdrawn & Lead Factor \\
\hline \multirow{5}{*}{ Surveillance Chain } & $\mathrm{A}$ & $37^{\circ}$ & $2862 \mathrm{~mm}$ & {$[-1190,2890] \mathrm{mm}$} & July 1984 & 0.60 \\
\cline { 2 - 7 } & $\mathrm{B}$ & $76^{\circ}$ & $2862 \mathrm{~mm}$ & {$[-1190,2890] \mathrm{mm}$} & July 1999 & 1.22 \\
\cline { 2 - 7 } & $\mathrm{C}$ & $127^{\circ}$ & $2862 \mathrm{~mm}$ & {$[-1190,2890] \mathrm{mm}$} & N/A & 0.60 \\
\cline { 2 - 7 } & $\mathrm{D}$ & $217^{\circ}$ & $2862 \mathrm{~mm}$ & {$[-1190,2890] \mathrm{mm}$} & N/A & 0.60 \\
\cline { 2 - 7 } & $\mathrm{E}$ & $256^{\circ}$ & $2862 \mathrm{~mm}$ & {$[-1190,2890] \mathrm{mm}$} & N/A & 1.21 \\
\cline { 2 - 7 } & $\mathrm{F}$ & $307^{\circ}$ & $2862 \mathrm{~mm}$ & {$[-1190,2890] \mathrm{mm}$} & N/A & 0.60 \\
\cline { 2 - 7 } & $\mathrm{G}$ & $346^{\circ}$ & $2862 \mathrm{~mm}$ & {$[-1190,2890] \mathrm{mm}$} & N/A & 1.21 \\
\cline { 2 - 7 } & $\mathrm{H}$ & $162^{\circ}$ & $2565 \mathrm{~mm}$ & {$[-170,1870] \mathrm{mm}$} & July 1984 & 20.46 \\
\cline { 2 - 7 } & $\mathrm{K}$ & $53^{\circ}$ & $2413 \mathrm{~mm}$ & {$[-170,1870] \mathrm{mm}$} & July 1984 & 0.35 \\
\cline { 2 - 7 } & $\mathrm{L}$ & $127^{\circ}$ & $2413 \mathrm{~mm}$ & {$[-170,1870] \mathrm{mm}$} & April 1997 & 0.35 \\
\cline { 2 - 7 } & $\mathrm{M}$ & $233^{\circ}$ & $2413 \mathrm{~mm}$ & {$[-170,1870] \mathrm{mm}$} & N/A & 0.34 \\
\hline \multirow{3}{*}{ Upper Core Grid } & $\mathrm{DE} / 39$ & $243^{\circ}$ & $336 \mathrm{~mm}$ & $2347 \mathrm{~mm}$ & August 1988 & N/A \\
\cline { 2 - 7 } & $\mathrm{DC} / 53$ & $350^{\circ}$ & $910 \mathrm{~mm}$ & $2347 \mathrm{~mm}$ & August 1988 & N/A \\
\cline { 2 - 7 } & $\mathrm{DC} / 69$ & $356^{\circ}$ & $2099 \mathrm{~mm}$ & $2347 \mathrm{~mm}$ & August 1988 & N/A \\
\hline \multirow{3}{*}{ Wide Range Neutron } & $\mathrm{N} 1$ & $225^{\circ}$ & $649 \mathrm{~mm}$ & $-1840 \mathrm{~mm}$ & August 2007 & N/A \\
\cline { 2 - 7 } & $\mathrm{N} 2$ & $225^{\circ}$ & $649 \mathrm{~mm}$ & $-1340 \mathrm{~mm}$ & August 2007 & N/A \\
\cline { 2 - 7 } & $\mathrm{M} 1$ & $225^{\circ}$ & $649 \mathrm{~mm}$ & $250 \mathrm{~mm}$ & August 2007 & N/A \\
\cline { 2 - 7 } & $\mathrm{O} 1$ & $225^{\circ}$ & $649 \mathrm{~mm}$ & $1340 \mathrm{~mm}$ & August 2007 & \\
\hline
\end{tabular}

design life time of the power plant. Instead, a Periodic Safety Report (PSR) is issued every ten years to the regulatory authority to review. If the regulatory authority is satisfied with the analysis, then the power plant will get approval for operation for an additional ten years.

Since the start of commercial operation, dosimetry and material samples from five surveillance chains have been analyzed. These in-vessel dosimetry evaluations were then used to validate the calculational models that were applied in the plant-specific neutron transport analysis. In addition, retrospective dosimetry analyses were performed on an in-core wide range neutron monitor tube and on upper core grid material samples. In May 2013, ex-vessel neutron dosimetry was installed in the annular gap between the RPV insulation and the sacrificial concrete bioshield. This dosimetry was withdrawn in April 2014 and its analysis will be the subject of a future paper.

\section{Surveillance Chains \& Retrospective Dosimetry}

The Ringhals Unit 1 reactor has an extensive RPV materials surveillance program in place with eleven in-vessel surveillance chains containing material samples and dosimetry. The chains are constructed from a variety of links which hold material samples and/or dosimeters. Eight of the surveillance chains (A, B, C, D, E, F, G, and H) are loaded with carbon steel material samples representative of the RPV material whereas three of the chains $(\mathrm{K}, \mathrm{L}$, and $\mathrm{M}$ ) are loaded with stainless steel material samples representative of the moderator tank material. The chains are mounted on either the outside (Chains A, B, C, D, E, F, G, and H) or inside (Chains K, L, and M) of the moderator tank wall. Because the chains are segmented, attention must be paid to the axial position of the dosimetry samples within the chains during the analysis. A summary of the surveillance chains is given in Table 1 with as-designed positions specified. While minor variations are expected between the as-irradiated and as-designed positions, the samples are precisely located and the effect of this variation is deemed negligible.

When discussing irradiated material samples, it is valuable to understand the lead factor associated with the samples. The lead factor applicable to any given operating period is defined as the ratio of the neutron $(\mathrm{E}>1.0 \mathrm{MeV})$ fluence accrued by the test specimens contained at the core midplane elevation within the surveillance chain to the corresponding neutron $(\mathrm{E}>1.0 \mathrm{MeV})$ fluence accrued at the location of maximum exposure at the RPV clad/base metal interface. Surveillance Chains K, L, and M contain stainless steel moderator tank material samples. Therefore, the lead factors for those three chains are calculated relative to the maximum moderator tank wall fluence. The lead factors at present or at the time of withdrawal, as applicable, are also shown in Table 1. 
In addition to the traditional reactor dosimetry contained in the surveillance chains as described previously, in August 1988 (after 8.7 EFPY of operation) the upper core grid was replaced. At that time, three material samples were taken from different locations within the same octant of the upper core grid to act as retrospective dosimetry. These samples were then processed and the thermal ${ }^{59} \mathrm{Co}(\mathrm{n}, \gamma){ }^{60} \mathrm{Co}$ activity levels were reported. In addition, samples were taken to characterize the material properties.

Also, a wide range neutron monitor (WRNM) tube was inserted in September 1987 and removed in August 2007 (a total of 14.4 EFPY of irradiation). Following irradiation, the WRNM tube was cut into three $500 \mathrm{~mm}$ segments and four total samples were taken from these segments to act as another form of retrospective dosimetry. Details regarding the segment spacing and locations are unavailable, but the three segments roughly correspond to core inlet, core midplane, and core exit. The material samples were taken from the top and bottom of the core inlet sample, the top of the core midplane sample, and the bottom of the core exit sample. Each sample was analyzed and the fast ${ }^{54} \mathrm{Fe}(\mathrm{n}, \mathrm{p}){ }^{54} \mathrm{Mn}$ reaction rates and the thermal ${ }^{59} \mathrm{Co}(\mathrm{n}, \gamma){ }^{60} \mathrm{Co}$ activity levels were reported. A summary of the retrospective dosimetry samples analyzed to date is also given in Table 1.

Elemental compositions for both sets of retrospective dosimetry were also reported. However, the pedigree of the composition data is unclear and will be discussed later when comparing calculations and measurements.

\section{RAPTOR-M3G Model for Neutron Transport Calculations}

The RAPTOR-M3G code is a Westinghouse-developed three-dimensional discrete ordinates radiation transport theory code that is written to operate in parallel on multiple processors. RAPTOR-M3G performs calculations in X-Y-Z or R- $\Theta-Z$ geometries $[1,2]$.

The RAPTOR-M3G model for the Ringhals Unit 1 reactor is a 1/4-core model that extended axially over $9000 \mathrm{~mm}$ and radially out to $4000 \mathrm{~mm}$. Axially, the active fuel covers the central portion of the model between $\pm 1840 \mathrm{~mm}$. The overall RAPTOR-M3G model includes approximately $22.6 \mathrm{M}$ mesh (335 radial, 225 azimuthal, 300 axial). Because of the significant variation in coolant density and void fraction throughout the fuel region, the fuel is evenly split into twelve zones axially and bundle-wise radially leading to 1944 unique fuel zones. The remaining reactor geometry accounts for an additional 36 zones. Two elevation views of the RAPTOR-M3G geometry are shown in Fig. 1. The calculations use the BUGLE-96 cross-section library [3]. The ENDF/B-VI-based BUGLE-96 library is a 67 energy group (47 neutron, 20 gamma ray) coupled cross-section set produced specifically for light-water reactor applications. This cross-section data is used to create smeared materials on a zone-wise basis, including each of the fuel zones. Using this approach, the local coolant density and void fraction behavior (both inter- and intra-bundle) can be accurately represented. Elsewhere within the RPV, system operating temperatures are treated on a fuel cycle-specific basis. In the transport analysis, anisotropic scattering is treated with a $\mathrm{P}_{3}$ expansion of the cross-sections and angular discretization is modelled with an $\mathrm{S}_{8}$ angular quadrature. A directional-theta-weighted differencing scheme was used [4]. An energyand space-dependent fixed distributed source based on fuel cycle-specific core power, burnup, and enrichment distributions is used. This accounts for the mix of fissioning isotopes $\left({ }^{235} \mathrm{U},{ }^{238} \mathrm{U},{ }^{239} \mathrm{Pu}\right.$, ${ }^{240} \mathrm{Pu},{ }^{241} \mathrm{Pu}$, and ${ }^{242} \mathrm{Pu}$ ) on an assembly-wise basis. Pin-by-pin relative power distributions are used for all of the peripheral fuel assemblies (those assemblies with a face or corner in contact with the moderator tank baffle plates). A typical RAPTOR-M3G model, as described previously, runs for $\sim 11$ hours on 60 processors. Each RAPTOR-M3G calculation produces a global solution for neutron and gamma ray distributions as a function of energy and space to which responses functions can be applied to derive quantities of interest. As an example, Fig. 2 shows a typical neutron $(\mathrm{E}>1.0 \mathrm{MeV})$ flux distribution on the moderator tank and RPV. 


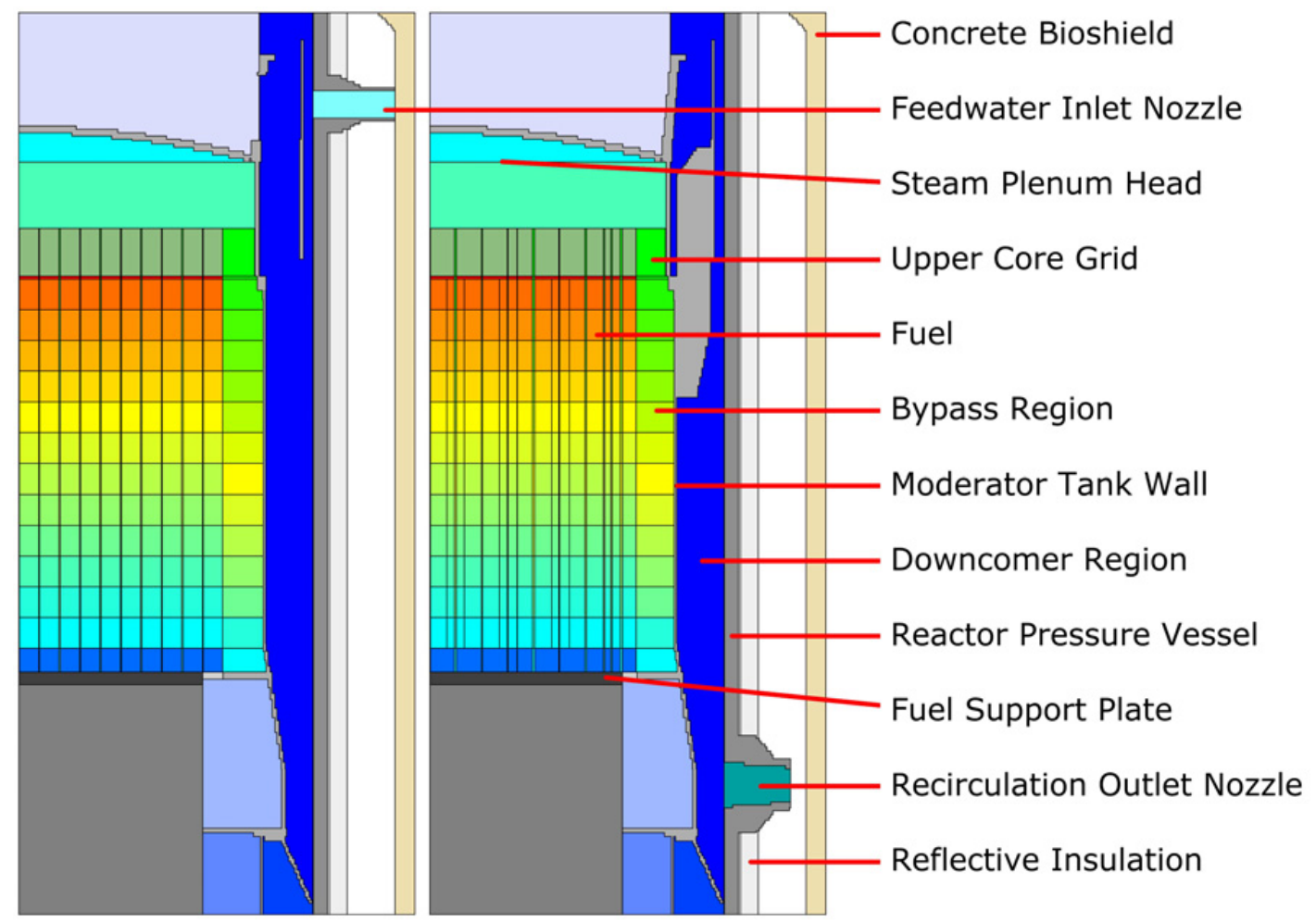

Figure 1. Elevation Views of Geometry Showing Feedwater Inlet and Recirculation Outlet Nozzles.

\section{Least-Squares Evaluation of Dosimetry}

The least-squares evaluation of the dosimetry sets use the FERRET code [5] to combine the results of the plant-specific neutron transport calculations and the sensor reaction rate measurements to determine best-estimate values of fast neutron exposure parameters, e.g., neutron fluence rate (E $>1.0 \mathrm{MeV}$ and $\mathrm{E}>0.1 \mathrm{MeV})$ and iron atom displacement rate (dpa/s) along with the associated uncertainties. The application of the least-squares methodology requires the following input:

- The calculated neutron energy spectrum and associated uncertainties at the measurement location(s),

- The measured reaction rates and associated uncertainty at the measurement location(s), and

- The energy-dependent dosimetry reaction cross-sections and associated uncertainties for each sensor contained in the multiple foil sensor sets.

The calculated neutron energy spectrum is obtained from the results of the plant- and cycle-specific RAPTOR-M3G neutron transport calculations described previously. The sensor reaction rates are derived from the measured activities for each dosimetry set analyzed. The dosimetry reaction crosssections and uncertainties are obtained from the ENDF/B-VI-based SNLRML dosimetry cross-section library [6]. It should be noted that this approach is consistent with and meets the requirements of Regulatory Guide 1.190 [7]. 


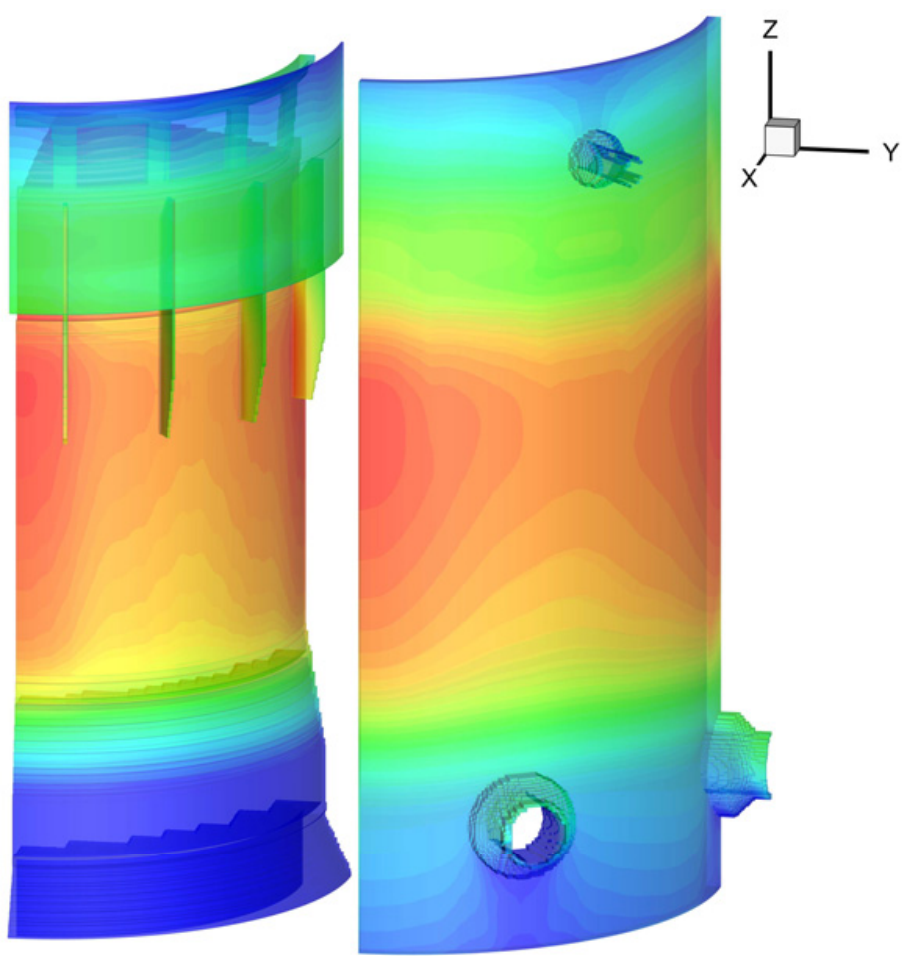

Figure 2. Neutron $(\mathrm{E}>1.0 \mathrm{MeV})$ Flux Distribution Throughout the Moderator Tank (Left) and RPV (Right).

\section{Comparisons of Calculations and Measurements}

A summary of the Ringhals Unit 1 surveillance chain analysis and best estimate evaluation results is given in Table 2.

The results show the comparison of fast reaction measured to calculated results without recourse to the least-squares adjustment procedure. For this analysis, each dosimeter was analyzed with regard to its explicit elevation at a given radius as shown in Table 1. The results of these individual calculations are then compared with the corresponding measured reaction rates and the overall comparisons averaged. It should be noted that for the surveillance chains, sufficient time had elapsed between the withdrawal of the dosimetry and the analysis that the ${ }^{63} \mathrm{Cu}(\mathrm{n}, \alpha){ }^{60} \mathrm{Co}$ was the only fast reaction capable of producing reliable results. The results from the dosimetry evaluations of Chain $\mathrm{K}$ have been discarded. The results were discarded because of significant inconsistencies between the results for that chain and the remaining sensor sets from the other four chains as well as the poor fit of the data quantified by the $\chi^{2} /$ degrees-of-freedom value significantly larger than 1.0.

Next, best estimate to calculation $(\mathrm{BE} / \mathrm{C})$ ratios for neutron $(\mathrm{E}>1.0 \mathrm{MeV})$ flux and iron atom displacement rate resulting from the least-squares evaluation of each dosimetry set, neglecting the ${ }^{59} \mathrm{Co}(\mathrm{n}, \gamma){ }^{60} \mathrm{Co}$ reaction, are provided for the in-vessel irradiations. The cobalt reaction is neglected for two reasons. First, fast neutrons are generally of greater importance when considering radiation damaging effects on steel structures. Second, the ${ }^{59} \operatorname{Co}(\mathrm{n}, \gamma){ }^{60}$ Co reaction consistently shows historically poor agreement between measured and calculated ratios because of the heavy dependence on a wellresolved thermal flux region (a known weakness with current LWR shielding cross-section libraries). For the in-vessel chains, the average BE/C ratio is 1.10 with an associated uncertainty of $9.0 \%$ for neutron $(\mathrm{E}>1.0 \mathrm{MeV})$ flux and 1.10 with an associated uncertainty of $8.9 \%$ for the iron atom displacement rate. 
Table 2. Ringhals Unit 1 Surveillance Chain Analysis Summary.

\begin{tabular}{|c|c|c|c|c|c|c|}
\hline \multirow{2}{*}{ Chain } & \multirow{2}{*}{$\begin{array}{c}\text { Dosimeters } \\
\text { Analyzed }\end{array}$} & \multirow{2}{*}{$\underset{{ }^{63} \mathrm{Cu}(\mathbf{C}, \alpha)}{\text { M/C Ratio }}$} & \multicolumn{2}{|c|}{ Flux $(E>1.0 \mathrm{MeV})$} & \multicolumn{2}{|c|}{ dpa/s } \\
\hline & & & $\mathrm{BE} / \mathrm{C}$ & $\%$ std dev & $\mathrm{BE} / \mathrm{C}$ & $\%$ std dev \\
\hline A & 13 & 1.16 & 1.13 & 11 & 1.13 & 11 \\
\hline B & 3 & 1.28 & 1.22 & 11 & 1.22 & 10 \\
\hline $\mathrm{H}$ & 3 & 0.99 & 0.99 & 2 & 0.99 & 2 \\
\hline $\mathrm{K}$ & 2 & - & - & - & - & - \\
\hline $\mathrm{L}$ & 1 & 1.08 & 1.06 & - & 1.06 & - \\
\hline \multicolumn{2}{|c|}{ Average } & 1.13 & 1.10 & 9.0 & 1.10 & 8.9 \\
\hline
\end{tabular}

The M/C data sets listed as well as the $\mathrm{BE} / \mathrm{C}$ data sets provide a validation of the plant-specific neutron transport calculations. Each of these data comparisons shows that for in-vessel locations, the aggregate measurements and calculations agree well within the $20 \%$ criterion specified in Regulatory Guide 1.190 and within the $13 \%(1 \sigma)$ uncertainty typically assigned to the absolute transport calculations [8].

Summaries of the measured to calculation (M/C) ratios are not listed for either the upper core grid or the wide range neutron monitor tube samples. The disagreement between the measured and calculated values is such that an average value is not beneficial. However, some notable features are observed regarding the individual values.

First, in reviewing the ${ }^{59} \mathrm{Co}(\mathrm{n}, \gamma){ }^{60} \mathrm{Co}$ reaction data for the upper core grid, it is recognized that the $\mathrm{M} / \mathrm{C}$ ratios for each sample agree well (with $6 \% 1 \sigma$ standard deviation). Therefore, it is expected that a systematic factor is causing the disagreement between the measured and calculated values. Such factors might include: axial core power distribution inaccuracy, inappropriate dosimetry processing (e.g., inappropriate spatial correction or inappropriate decay correction), inaccurate reporting of the elemental composition, or other material impurities affecting the measured activity. Regarding the elemental composition, investigating the variation needed to produce acceptable $\mathrm{M} / \mathrm{C}$ values indicated that minimal perturbation on the ${ }^{59} \mathrm{Co}$ abundance was needed, and was well within the typical range of reactor-internals grade stainless steel, to produce acceptable $\mathrm{M} / \mathrm{C}$ values.

Also, in reviewing both the ${ }^{54} \mathrm{Fe}(\mathrm{n}, \mathrm{p}){ }^{54} \mathrm{Mn}$ and the ${ }^{59} \mathrm{Co}(\mathrm{n}, \gamma){ }^{60} \mathrm{Co}$ reaction data for the wide range neutron monitor tubes, the $\mathrm{M} / \mathrm{C}$ ratios agree to $43 \%$ and $32 \%(1-\sigma)$ for the ${ }^{54} \mathrm{Fe}(\mathrm{n}, \mathrm{p})^{54} \mathrm{Mn}$ and ${ }^{59} \mathrm{Co}(\mathrm{n}, \gamma){ }^{60} \mathrm{Co}$ reactions, respectively. The agreement improves to $22 \%(1-\sigma)$ for each reaction when Sample N1 is neglected. This would suggest that Sample N1 is behaving inconsistently with the remaining samples. This is further confirmed by the $\chi^{2} /$ degrees-of-freedom value significantly larger than 1.0 for Sample N1 unlike the other three samples. Since Sample N1 is located nearest the bottom of the core, where the axial core power distribution varies the most, the uncertainty in the position of the sample can explain the inconsistent behavior. Regardless, additional factors that would adversely affect the M/C comparison are the same as those described for the upper core grid, namely: axial core power distribution inaccuracy, inappropriate dosimetry processing (e.g., inappropriate spatial correction or inappropriate decay correction), inaccurate reporting of the elemental composition, or other material impurities affecting the measured activity. It is hoped that when retrospective dosimetry is retrieved in the future, material composition can be more precisely characterized and axial power distribution and decay corrections can be more robustly accounted for. 


\section{$15^{\text {th }}$ ISRD}

\section{Future Ringhals Unit 1 Surveillance Program Plans}

As part of the continued operational plan for Ringhals Unit 1, Surveillance Chain C will be extracted during the Summer 2014 outage and later analyzed at a hot cell laboratory. The results of the analysis are expected to be available in Summer 2015. That will bring the total number of analyzed surveillance chains containing RPV material to four: three standard chains $(\mathrm{A}-\mathrm{C})$ and one accelerated chain $(\mathrm{H})$. The number of evaluated chains and the fact that they are evenly distributed throughout the fluence lifetime of the reactor gives a well-defined data set which provides confidence in the evaluation of the mechanical properties of the RPV. At present, the operational plan for Ringhals Unit 1 is for a total of 50 years. The accelerated Chain $\mathrm{H}$ is representative of RPV material properties well beyond 50 years of operation. As such, the next planned withdrawal of a surveillance chain is uncertain but is expected to occur in 2024. That will likely be the last surveillance chain to be withdrawn from Ringhals Unit 1 . In the meantime, it is planned to evaluate the ex-vessel neutron dosimetry every five years to perform continuous RPV neutron exposure monitoring, with the next withdrawal scheduled for 2019.

\section{Summary}

The plant- and cycle-specific lifetime neutron fluence analysis of the Ringhals Unit 1 boiling water reactor was successful in that in-vessel dosimetry withdrawn to date has been analyzed and shows good agreement with calculated results with the exception of Chain $\mathrm{K}$ (which is regarded as an outlier). In the future, there are plans to analyze the dosimetry sooner after withdrawal (as noted in Sect. 7) in order to have the highest fidelity dosimetry counting results possible. Nevertheless, these measurements to date validate the calculations made for the fluence environment in and around the RPV and moderator tank and can thus be used to predict future irradiation behavior with continued periodic validation with dosimetry measurements.

The retrospective dosimetry sample analysis met with mixed success. For the upper core grid, only the ${ }^{59} \mathrm{Co}(\mathrm{n}, \gamma){ }^{60} \mathrm{Co}$ reaction information is available. As a thermal reaction, this is known to be difficult to characterize. Regardless, the calculated values show a consistent bias versus the measurements which suggests a systematic issue. Further, minor yet reasonable perturbations on the cobalt impurity level yield calculated results that show good agreement with measurements. Given the good comparison between the in-vessel dosimetry and calculations, the likeliest cause of the upper core grid measurement disagreement is an inaccurate material composition characterization. There were similar issues with the wide range neutron monitor tube and it is hoped that in the future the material composition characterization of retrospective dosimetry samples will provide data with a clearer pedigree.

\section{References}

[1] G. Longoni and S. L. Anderson, Reactor Dosimetry Applications Using RAPTOR-M3G: A New Parallel 3-D Radiation Transport Code. 2009. Reactor Dosimetry State of the Art 2008 - Proceedings of the 13th International Symposium on Reactor Dosimetry. World Scientific. Singapore

[2] M. A. Hunter et al., Extension of RAPTOR-M3G to R- $\Theta-Z$ Geometry For Use In Reactor Dosimetry Applications. 2009. Reactor Dosimetry State of the Art 2008 - Proceedings of the 13th International Symposium on Reactor Dosimetry. World Scientific. Singapore

[3] ORNL RSICC DLC-185, BUGLE-96 - Coupled 47 Neutron, 20 Gamma-Ray Group Cross Section Library Derived from ENDF/B-VI for LWR Shielding and Pressure Vessel Dosimetry Applications. March 1996

[4] B. Petrović and A. Haghighat, New Directional Theta-Weighted (DTW) Differencing Scheme and Reduction of Estimated Pressure Vessel Fluence Uncertainty. 1998. Proceedings of the 9th International Symposium on Reactor Dosimetry. World Scientific. Singapore 
[5] ORNL RSICC PSR-145, FERRET - Least Squares Solution to Nuclear Data and Reactor Physics Problems. January 1980

[6] ORNL RSICC DLC-178, SNLRML - Recommended Dosimetry Cross Section Compendium. July 1994

[7] USNRC Regulatory Guide 1.190, Calculational and Dosimetry Methods for Determining Pressure Vessel Neutron Fluence. March 2001

[8] J. D. Andracheck et al., WCAP-14040-A, Revision 4, Methodology Used to Develop Cold Overpressure Mitigating System Setpoints and RCS Heatup and Cooldown Limit Curves. May 2004 